Corresponding author: gholsonjlyon@gmail.com

(c) 2016 Malcolmson et al. This article is distributed under the terms of the Creative Commons Attribution License, which permits unrestricted reuse and redistribution provided that the original author and source are credited.

Ontology terms: absent speech; action tremor; appendicular hypotonia; blepharospasm; broad forehead; bulbar palsy; developmental regression; developmental stagnation at onset of seizures; dysphagia; epileptic encephalopathy; exaggerated startle response; failure to thrive in infancy; full cheeks; gastroesophageal reflux; gastrostomy tube feeding in infancy; generalized tonic seizures; generalized tonic-clonic seizures on awakening; gingival overgrowth; hypoxemia; infantile axial hypotonia; intellectual disability, severe; neck muscle weakness; no social interaction; obstructive sleep apnea; respiratory difficulties

Published by Cold Spring Harbor Laboratory Press

doi: $10.1101 / \mathrm{mcs} . a 001073$

\section{SCN8A mutation in a child presenting with seizures and developmental delays}

\author{
Janet Malcolmson, ${ }^{1,2,8}$ Robert Kleyner, ${ }^{1,8}$ David Tegay, ${ }^{1}$ Whit Adams, ${ }^{3}$ \\ Kenneth Ward, ${ }^{4}$ Justine Coppinger, ${ }^{4}$ Lesa Nelson, ${ }^{4}$ Miriam H. Meisler, ${ }^{5}$ \\ Kai Wang, ${ }^{3,6,7}$ Reid Robison, ${ }^{3}$ and Gholson J. Lyon ${ }^{1,3}$ \\ ${ }^{1}$ Stanley Institute for Cognitive Genomics, Cold Spring Harbor Laboratory, Cold Spring Harbor, New York \\ 11724, USA; ${ }^{2}$ Genetic Counseling Graduate Program, Long Island University (LIU), Brookville, New York \\ 11548, USA; ${ }^{3}$ Utah Foundation for Biomedical Research, Salt Lake City, Utah 84107, USA; ${ }^{4}$ Affiliated Genetics, \\ Salt Lake City, Utah 84109, USA; ${ }^{5}$ Department of Human Genetics, University of Michigan, Ann Arbor, \\ Michigan 48109-5618, USA; ${ }^{6}$ Zilkha Neurogenetic Institute, University of Southern California, Los Angeles, \\ California 90089, USA; ${ }^{7}$ Department of Psychiatry and Behavioral Sciences, Keck School of Medicine, \\ University of Southern California, Los Angeles, California 90033, USA
}

Abstract The SCN8A gene encodes the sodium voltage-gated channel alpha subunit 8. Mutations in this gene have been associated with early infantile epileptic encephalopathy type 13. With the use of whole-exome sequencing, a de novo missense mutation in SCN8A was identified in a 4-yr-old female who initially exhibited symptoms of epilepsy at the age of 5 mo that progressed to a severe condition with very little movement, including being unable to sit or walk on her own.

[Supplemental material is available for this article.]

\section{INTRODUCTION}

Mutations in SCN8A are associated with cognitive impairment with or without cerebellar ataxia (OMIM\#613406) and with early infantile epileptic encephalopathy-13 (EIEE13, OMIM\#614558). Loss-of-function mutations can be associated with cerebellar ataxia and cognitive issues, whereas gain-of-function mutations can underlie epileptic encephalopathy (O'Brien and Meisler 2013). De novo mutations in SCN8A have been discovered through genome and exome sequencing, and SCN8A can now be evaluated with commercial epilepsy panels.

Epileptic encephalopathy is characterized by seizure activity that progresses to cerebral dysfunction leading to severe cognitive, motor, and behavioral impairments (Vaher et al. 2014). Approximately $1 \%$ of early infantile epileptic encephalopathies are associated with missense mutations in the SCN8A gene, and approximately 50 cases have been described in the literature (Veeramah et al. 2012; Larsen et al. 2015; Wagnon and Meisler 2015; Meisler et al. 2016). In a few cases, the mutation was inherited from a mosaic parent, but the majority of disease-contributory mutations are de novo missense mutations (Wagnon and Meisler 2015). EIEE13 has an average age of onset between 3 and 7 mo, and individuals present with various types of seizures, including tonic-clonic, generalized tonic, atonic, myoclonic, and focal and absence seizures, whereas febrile seizures are rare (Ohba et al. 2014). There

\footnotetext{
${ }^{8}$ These authors contributed equally to this work.
} 
is often developmental regression, and movement disorders are present with $50 \%$ of affected individuals unable to sit or walk (Meisler et al. 2016).

SCN8A is located on Chromosome 12q13 and encodes the sodium voltage-gated channel alpha subunit (also know as $\mathrm{Na}_{v} 1.6$ ), which functions in the rapid depolarization of sodium channels during generation of action potentials in neurons. $\mathrm{Na}_{v} 1.6$ is one of the three major sodium channels in the brain and is involved in the regulation and propagation of firing patterns of excitatory and inhibitory neurons (O'Brien and Meisler 2013). $\mathrm{Na}_{v} 1.6$ is localized to the axonal initial segment and to nodes of Ranvier in myelinated neurons. Mutations in the related sodium channel gene SCN1A contribute to Dravet syndrome, and mutations in SCN2A contribute to Ohtahara syndrome (Wagnon and Meisler 2015).

Ten patient mutations of SCN8A have been evaluated with functional tests in transfected cells (Veeramah et al. 2012; de Kovel et al. 2014; Estacion et al. 2014; Blanchard et al. 2015; Wagnon et al. 2016). In eight of the 10 cases, gain-of-function effects were observed. The most common defect is impaired channel inactivation, which leads to a persistent sodium current and increased neuronal activity. In two cases, a hyperpolarizing shift in voltage dependence of channel activation was observed, also leading to hyperactivity. This gain-offunction mechanism is opposite to the situation in Dravet syndrome, where loss-of-function mutations in SCN1A are most common (Meisler et al. 2016).

Through advances in DNA sequencing technology, DNA can now be sequenced quickly and relatively inexpensively. Although such analyses focus on $\sim 1 \%$ of the human genome, this, nonetheless, still identifies tens of thousands of variants to analyze ( $O^{\prime}$ Rawe et al. 2013, 2015; Lyon and O'Rawe 2015). Through the use of existing and novel bioinformatics tools, we developed a protocol focused on identifying uncommon and deleterious mutations and prioritizing them by phenotypic relevance. We present here a detailed case study of a proband in which a de novo likely disease-contributing mutation in SCN8A was discovered. This study focused on the use of next-generation exome sequencing to find mutations by analyzing primarily protein-coding regions of the DNA.

\section{RESULTS}

\section{Clinical Presentation and Family History}

The proband is a 4-yr-old female presenting with idiopathic epilepsy (10 to 15 seizures per day), cortical blindness, and developmental regression (Figs. 1 and 2; Supplemental Video 1). Her medical records show that she has no language or motor skills, is fed through a $G$ tube, and has recurrent fevers and osteopenia. She had acute fractures of her distal radius and distal ulna bilaterally as well as on the left distal tibia and left distal fibula while on a ketogenic diet. Table 1 outlines her phenotypic features using Human Phenotype Ontology (HPO) terms. Before whole-exome sequencing (WES), an infantile epileptic encephalopathy

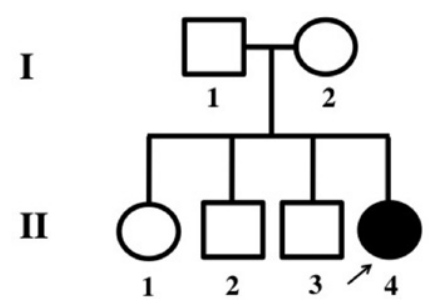

Figure 1. Pedigree: II-4, the affected proband (4-yr-old), is the daughter of an unaffected, nonconsanguineous couple. The proband has one older (14-yr-old) unaffected sister and two older (11-yr-old and 7-yr-old) unaffected brothers. 

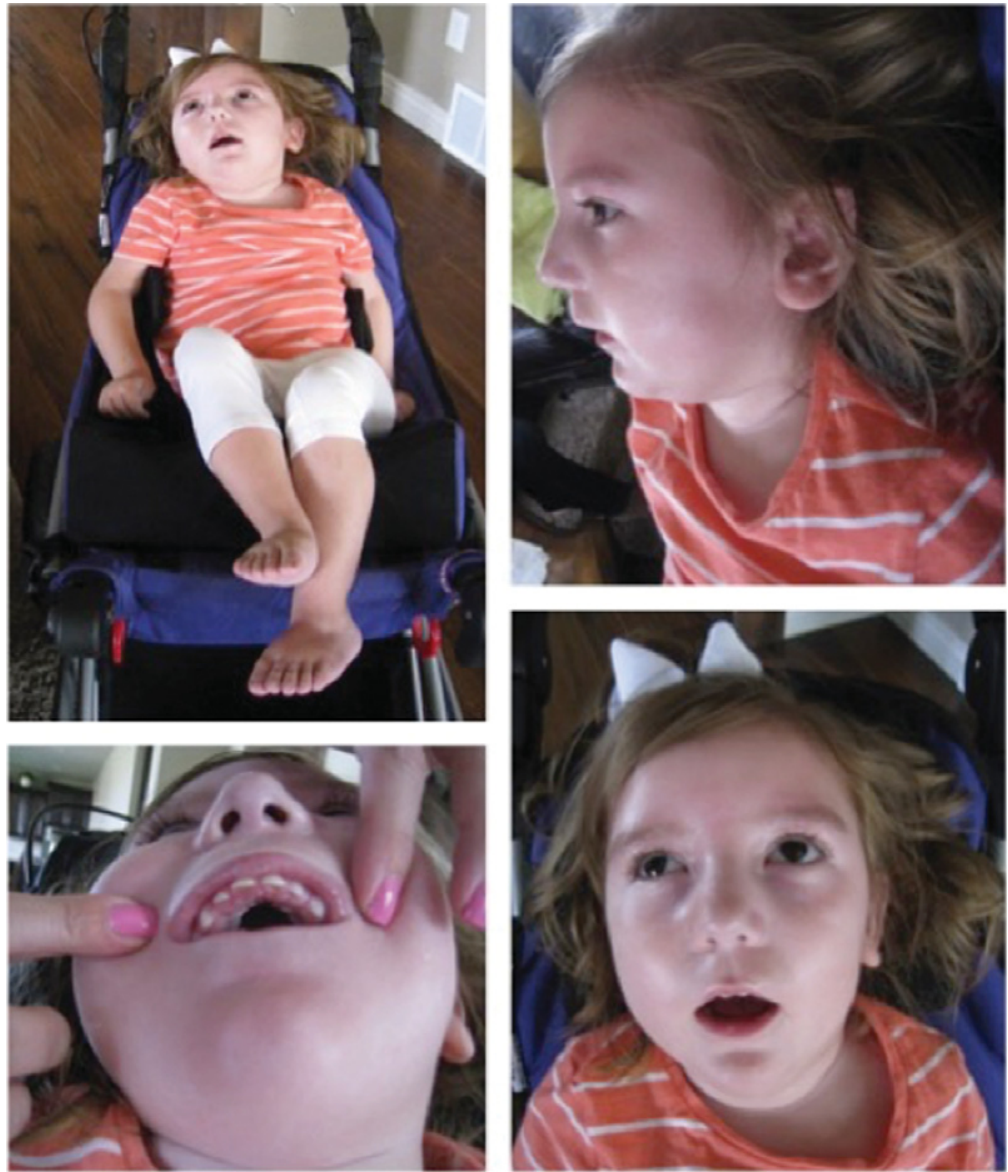

Figure 2. Pictures of phenotype of proband. Facial features include brachycephaly, broad forehead, broad nasal root, hypoplastic alae nasi, full cheeks, gingival hyperplasia, mild micrognathia, and hypotonic facies.

panel that did not include SCN8A was ordered for this proband. The results showed a variant of uncertain significance in exon 3 of the GRIN2A gene that encodes the glutamate ionotropic receptor $\mathrm{N}$-methyl-D-aspartate (NMDA)-type subunit 2A, resulting in an amino acid change from phenylalanine to isoleucine at position 183. Parents of the proband were tested for this variant and the unaffected father was found to carry this variant. This proband had a high-resolution whole-genome single-nucleotide polymorphism (SNP)/copy-number microarray analysis using the Cytoscan HD platform, and Chromosome Analysis Suite Software (Affymetrix) was used for analysis. The result was normal and no clinically significant copynumber variants were discovered.

The only reported complication during pregnancy was a group B streptococcal infection in the mother. From birth to 3 mo of age, the proband met all milestones, although her parents report she was slightly hypotonic. At 3 mo, she experienced her first seizure-like activity, although medical tests revealed no abnormalities. The evening after her 5-mo vaccinations, including diphtheria (DT), Haemophilus influenzae type B (Hib) conjugate (PRP-T), inactivated polio vaccine (IPV), and pneumococcal conjugate vaccine (PCV13), she was admitted to the emergency department with increased seizure activity, nystagmus, hypotonia, and fever, although medical tests revealed no pertinent abnormalities. A direct link between the vaccination and the onset of symptoms has not been established, and a link is not proposed by the authors. 
Table 1. Summary of the clinical features in this proband

\begin{tabular}{|c|c|}
\hline Features (Human Phenotype Ontology ID) & Proband \\
\hline \multicolumn{2}{|l|}{ Epilepsy } \\
\hline Epileptic encephalopathy (HP:0200134) & + \\
\hline Generalized tonic seizures (HP:0010818) & + \\
\hline Generalized tonic-clonic seizures on awakening (HP:0007193) & + \\
\hline EEG abnormality (HP:0002353) & + \\
\hline \multicolumn{2}{|l|}{ Developmental/intellectual disability } \\
\hline Global developmental delay (HP:0001263) & + \\
\hline Developmental stagnation at onset of seizures (HP:0006834) & + \\
\hline Developmental regression (HP:0002376) & + \\
\hline Absent speech (HP:0001344) & + \\
\hline Intellectual disability, severe (HP:0010864) & + \\
\hline Motor delay (HP:0001270) & + \\
\hline \multicolumn{2}{|l|}{ Neurological } \\
\hline Generalized hypotonia (HP:0001290) & + \\
\hline Appendicular hypotonia (HP:0012389) & + \\
\hline Infantile axial hypotonia (HP:0009062) & + \\
\hline Neck muscle weakness (HP:0000467) & + \\
\hline Rigidity (HP:0002063) & + \\
\hline Spasticity (HP:0001257) & + \\
\hline \multicolumn{2}{|l|}{ Growth/feeding } \\
\hline Failure to thrive in infancy (HP:0001531) & + \\
\hline Gastrostomy tube feeding in infancy (HP:0011471) & + \\
\hline Gastroesophageal reflux (HP:0002020) & + \\
\hline Dysphagia (HP:0002015) & + \\
\hline \multicolumn{2}{|l|}{ Respiratory } \\
\hline Respiratory difficulties (HP:0002880) & + \\
\hline Hypoxemia (HP:0012418) & + \\
\hline Abnormality of the tonsils (HP:0100765) & + \\
\hline Obstructive sleep apnea (HP:0002870) & + \\
\hline \multicolumn{2}{|l|}{ Dysmorphism } \\
\hline Brachycephaly (HP:0000248) & + \\
\hline Broad forehead (HP:0000337) & + \\
\hline Broad nasal root or bridge (HP:0000431) & + \\
\hline Hypoplastic alae nasi (HP:0000430) & + \\
\hline Full cheeks (HP:0000293) & + \\
\hline Gingival overgrowth (HP:0000212) & + \\
\hline Micrognathia (mild) (HP:0000347) & + \\
\hline Hypotonic facies & + \\
\hline \multicolumn{2}{|l|}{ Neurological } \\
\hline Exaggerated startle response (HP:0002267) & + \\
\hline Action tremor (HP:0002345) & + \\
\hline Blepharospasm (HP:0000643) & + \\
\hline Bulbar palsy (HP:0001283) & + \\
\hline
\end{tabular}




\begin{tabular}{lc}
\hline Table 1. Continued & \\
\hline Features (Human Phenotype Ontology ID) & Proband \\
\hline Nystagmus (HP:0000639) & + \\
Miscellaneous & + \\
Hyperreflexia (HP:0001347) & + \\
No social interaction (HP:0008763) & + \\
\hline
\end{tabular}

EEG, electroencephalograph.

An electroencephalogram (EEG) $2 \mathrm{wk}$ after the onset of severe symptoms revealed severe abnormalities, and the patient was diagnosed with idiopathic epileptic encephalopathy. The EEG showed the presence of frequent independent multifocal, mostly right posterior, epileptogenic abnormalities, consistent with a tendency toward partial onset seizures, and this is indicative of right hemispheric profound cerebral dysfunction. Follow-up EEGs showed multifocal sharp waves. Facial features include a short, upturned nose, full cheeks, a short philtrum, and a horizontal crease on the chin (Fig. 2). Magnetic resonance imaging (MRI) scans revealed no significant abnormalities that might contribute to the proband's condition; myelination was determined to be within normal range, and no congenital brain malformations or cytotoxic edema were found to suggest ischemia. The only abnormality found was a small, left periventricular cyst, most likely an incidental germinolytic cyst, which was determined to be unrelated to the proband's clinical presentation. Her physical manifestations include the absence of all mobility and motor coordination, with daily placement in a wheelchair, and the absence of a startle reflex, possibly secondary to neck weakness and poor eye control. The family history is unremarkable.

Medications that the proband is now taking include phenobarbital, carbamazepine, and Charlotte's Web (Hemp extract). The addition of carbamazepine has stopped her loop seizures and prevents her from having seizures as she enters and exits sleep. She has improved awareness, has more eye contact, and is able to shake a rattle.

\section{Genomic Analysis}

Blood samples from the proband, as well as blood and saliva samples from parents and siblings, were sequenced at Affiliated Genetics in Salt Lake City, Utah, where genomic DNA was extracted and exons sequenced using the Life Technologies Ampliseq Exome RDY kit and the Life Technologies Proton sequencing system (see Methods). These targeted regions were sequenced using the lon Proton sequencing system using lon $\mathrm{Hi}-\mathrm{O}$ Chemistry with 200-bp reads. The DNA sequence was compared with the University of California, Santa Cruz (UCSC) hg19 reference sequence, and several methods of analysis were applied to the sequence data (see Methods). A summary of variants called for all individuals in the family are described in Table 2, and coverage and mapping statistics are shown in Table 3. These analyses included in-house protocols, and several commercial software packages, including Tute Genomics, Omicia Opal, and Cartagenia v4.1, along with the use of the OTG-snpcaller pipeline (see Methods). In all analyses, we arrived at similar conclusions, but the various analyses helped to provide a more comprehensive and in-depth approach to the data. The Omicia results from three "QUAD" analyses (each with different siblings) are shown in Supplemental Tables 1-3. The scripts and programs created or rewritten by the author (R.K.) and used for analysis were uploaded to GitHub and can be found at https://github. com/rkleyner/PGM-WES-Pipeline.

As one example, for the OTG-snpcaller pipeline, the final variant call format (VCF) file for each individual contained 20,000 to 25,000 variants, of which 300 to 400 were found to be 
Table 2. Count of single-nucleotide polymorphisms (SNPs), insertions and deletions (indels), and the total number of variants for each individual sequenced

\begin{tabular}{lccc}
\hline Individual & Number of SNPs & Number of indels & Total number of variants \\
\hline Proband & 20,390 & 1756 & 22,146 \\
Mother & 20,889 & 1602 & 22,491 \\
Father & 20,705 & 1764 & 22,469 \\
Brother 1 & 21,199 & 1441 & 22,640 \\
Brother 2 & 21,302 & 1385 & 22,687 \\
Sister & 20,930 & 1705 & 22,635 \\
\hline
\end{tabular}

inherited. However, 2000 to 3000 variants were recognized as de novo, which is notably above the expected number of de novo mutations found in standard WES (Bamshad et al. 2011; Kong et al. 2012). In addition, even with an optimized variant calling pipeline, there were still a significant number of false positives. The number of variants found in the proband was 28,681 and the mean depth of coverage was 101-fold.

A GEMINI query (Paila et al. 2013) selected zero autosomal recessive mutations and 16 rare de novo mutations of interest. A unique de novo heterozygous variant in the SCN8A gene on Chromosome 12 at position 52,093,447 was identified (c.800T>C, p.Leu267Ser) (Table 4; Fig. 3A). This mutation is not present in any frequency in public databases such as Database for Short Genomic Variations (dbSNP) 137, 1000 Genomes phase 1 data, National Heart, Lung, and Blood Institute (NHLBI) 6500 exomes, or Exome Aggregation Consortium (ExAC) version 0.2 , which contains allelic information derived from approximately 60,000 exome sequences. The presence of the mutation was confirmed using Sanger sequencing following standard procedures (Fig. 3B). This missense mutation has not been previously identified in any other patient with epilepsy. Leucine 267 is located in transmembrane segment DIS5 of the sodium channel and is highly conserved through evolution (Fig. 4). The substitution changes the hydrophobic leucine residue to the hydrophilic serine, which is likely to alter channel function. The Combined Annotation-Dependent Depletion (CADD) score was 21.1 , indicating that this substitution is within the $0.78 \%$ of most deleterious mutations (99.2 percentile). The Sorting Intolerant from Tolerant (SIFT) ( $\mathrm{Ng}$ and Henikoff 2003) and Polymorphism Phenotyping (PolyPhen) scores (Adzhubei et al. 2013) were $<0.01$ and $>0.999$, respectively, also predicting that the mutation is deleterious. Analyses with Phenolyzer (Yang et al. 2015), wANNOVAR (Chang and Wang 2012), and PhenIX (Zemojtel et al. 2014) all predict that a heterozygous missense mutation in SCN8A is likely to contribute to the phenotype. The output from PhenIX, Phenolyzer, and WANNOVAR can be viewed in Supplemental Figures 1-3, respectively.

Table 3. Average read depth (exonic regions only), number of reads, and percent of reads mapped for each individual sequenced

\begin{tabular}{lccc}
\hline Individual & Average read depth & Number of reads & Reads mapped (\%) \\
\hline Proband & 99.32 & $36,808,143$ & 98.60 \\
Mother & 108.11 & $39,848,858$ & 98.78 \\
Father & 93.46 & $34,780,797$ & 98.39 \\
Brother 1 & 85.83 & $32,467,105$ & 97.47 \\
Brother 2 & 105.85 & $41,219,805$ & 96.64 \\
Sister & 125.08 & $45,749,335$ & 98.72 \\
\hline
\end{tabular}




\begin{tabular}{lllllll}
\hline \multicolumn{1}{l}{ Table 4. SCN8A variant } & & & & \\
\hline $\begin{array}{l}\text { Chr:position } \\
\text { GRCh37(hg19) }\end{array}$ & $\begin{array}{l}\text { HGVS } \\
\text { cDNA }\end{array}$ & $\begin{array}{c}\text { HGVS } \\
\text { protein }\end{array}$ & $\begin{array}{c}\text { Type of } \\
\text { variant }\end{array}$ & $\begin{array}{c}\text { Predicted } \\
\text { effect }\end{array}$ & $\begin{array}{c}\text { Genotype } \\
\text { origin }\end{array}$ \\
\hline 12:52,093,447 & c.800T>C & p.Leu267Ser & Substitution & Missense & Heterozygous & De novo \\
\hline
\end{tabular}

HGVS, Human Genome Variation Society.

The Online Mendelian Inheritance in Man (OMIM) database describes early infantile epileptic encephalopathy and cognitive impairment as two phenotypes associated with mutations in SCN8A. Because the proband has a similar phenotype, this is considered to be a mutation that contributes to the phenotype described here. Several studies have associated mutations in SCN8A with epilepsy, intellectual disability, and cranial features such as microcephaly (Veeramah et al. 2012; de Kovel et al. 2014; Estacion et al. 2014; Ohba et al. 2014; Vaher et al. 2014; Blanchard et al. 2015; Larsen et al. 2015; Wagnon and Meisler 2015).

\section{DISCUSSION}

Overall, the various analyses employed herein were able to efficiently and accurately detect variants in this family, uncovering a deleterious single-nucleotide variant (SNV) likely contributing to the severe phenotype and previously unidentified in clinical databases such as ClinVar and OMIM. By using existing databases to filter variants by rarity and deleteriousness, common variants and/or benign variants can be excluded from the analysis, leaving a small subset of variants to study and facilitating the analysis. Unlike other tools and protocols currently available, the described protocol efficiently examines the phenotypes associated with the genic location of a mutation by using phenotype analysis tools to efficiently provide a link between a variant and a set of phenotypes. This step prioritizes variants by phenotypic relevance quickly and efficiently, thus eliminating the need to manually research the phenotypes associated with many genes. WANNOVAR outputs were not depicted here

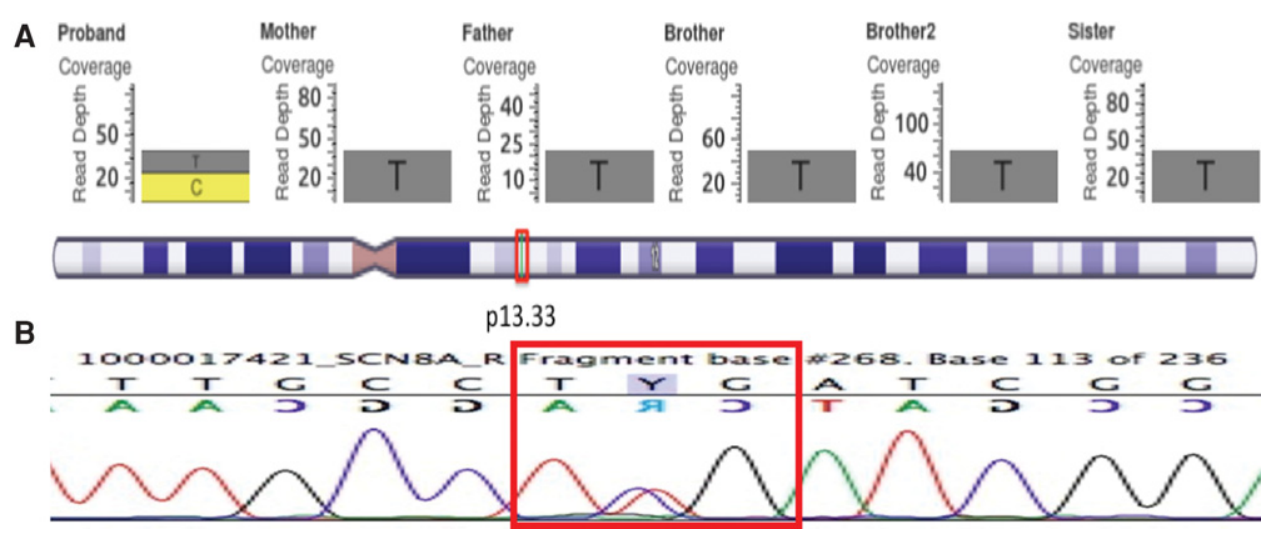

Figure 3. GenomeBrowse output for the mutation in SCN8A (A). The proband has a heterozygous $C$ substitution in Chromosome 12, position 52,093,447 in SCN8A. There are more than 20 reads covering the region, indicating that this is likely a true-positive mutation. None of the other family members appears to have this mutation, categorizing it as de novo. (B) Sanger sequencing for the proband confirms the presence of the mutation, as seen by the reverse (top) and forward (bottom) strands highlighted by the red box. 

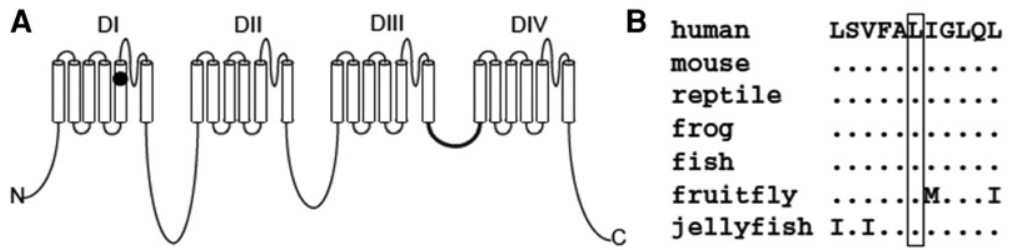

Figure 4. The de novo mutation Leu267Ser in SCN8A. Mammals and invertebrate sodium channel genes also have a conserved leucine in this location of the gene. (A) The site of the mutation is shown with a black circle. (B) This residue is highly conserved during evolution of vertebrate and invertebrate sodium channels.

because this relies on Phenolyzer to determine relationships to phenotype and was thus similar to the Phenolyzer output.

As in previous studies (Bragg et al. 2013b; Salipante et al. 2014), the lon Torrent Personal Genome Machine (PGM) sequencing results appeared to have several erroneous calls, especially around homopolymer regions and indels. These errors have been attributed to the PGM "dark sequencing" chemistry, which uses semiconductor chips to measure minute $\mathrm{pH}$ differences caused by the release of a hydrogen ion when a nucleotide attaches to a DNA template. In contrast, "light sequencing" platforms such as the Illumina MiSeq and HiSeq use high-resolution cameras or sensors to detect wavelengths of light emitted when a reaction occurs, such as when a fluorescently labeled nucleotide attaches to a prepared DNA template. Even with an optimized variant calling platform, the errors in PGM sequencing can lead to high false-positive rates. It is likely that the high number of de novo mutations, most of which have a low-quality score, were detected by the variant calling pipeline because of the small and sporadic occurrence of sequencing errors in homopolymer regions.

Although several mutations of SCN8A have been found to be associated with a similar syndrome (Wagnon and Meisler 2015), there is no entry in ClinVar for the p.Leu267Ser mutation. Interestingly, mutation of the corresponding leucine residue in SCN1A resulted in impaired channel inactivation (Kahlig et al. 2008), a common feature of SCN8A mutations in epileptic encephalopathy (Wagnon et al. 2016).

Although there are several factors leading to a susceptibility to fractures, including a ketogenic diet often recommended for seizures (Bergqvist et al. 2008), this patient exhibits co-occurrence of a mutation in SCN8A and bone fractures.

\section{METHODS}

\section{DNA Isolation and Sequencing}

Genomic DNA was extracted using standard methods (Puregene, QIAGEN). The Life Technologies Ampliseq Exome RDY kit (Thermo Fisher) was used to target the exon regions. Ninety-seven percent of Consensus Coding Sequences, with 5 bp exon padding, were amplified using 294,000 primer pairs. These products were sequenced using the Life Technologies Proton sequencing system with 200-bp reads using a P1V3 chip.

\section{Variant Calling}

The DNA sequence was aligned to the UCSC hg19 reference sequence and variants were called using the Torrent Suite software and the Torrent Variant caller. Only exonic variants and variants at the intron-exon boundary (one or two nucleotides into the intron and one nucleotide into the exon) were reviewed. For each variant considered, the depth of coverage was $\geq 10 \times$ and the quality score was $\geq 30$. Ethnicity and variant frequency were considered 
during analysis. Analysis of the variants was conducted by two independent reviews using inhouse protocols and two commercial software packages, Tute Genomics and Cartagenia v4.1. The variant in the SCN8A gene was confirmed by Sanger sequencing, which was completed using standard molecular biological methods as performed at Affiliated Genetics and/or the CSHL Sanger Sequencing Core Facility. American College of Medical Genetics and Genomics (ACMG) reporting criteria were also used to evaluate variants (Richards et al. 2015). The primers used in the Sanger sequencing procedure were as follows:

\section{SCN8A-800T>C_Forward 5'-TTCTGTCTCCTCAGGCCTGA-3' \\ SCN8A-800T>C - Reverse 5'-TGCAGAGAAGAGGCCACCTA-3'}

In additional analyses, binary alignment (BAM) files from the PGM platform were converted to FASTQ files. Variations in the sequenced DNA when compared with a reference genome (variants) were called for each family using the OTG-snpcaller pipeline, which has been reported to have a substantially higher proportion of sequencing reads mapped to a reference genome, lower false-positive rate, and overall lower error rate when analyzing sequences coming from the PGM platform (Zhu et al. 2014). Unlike other sequencing software and pipelines such as the Genome Analysis Toolkit (GATK) and FreeBayes (McKenna et al. 2010; Garrison and Marth 2012), OTG-snpcaller is specifically designed to take into account errors associated with PGM data, such as errors around homopolymers, thus increasing the overall accuracy. Variants were aligned to the hg19 assembly. A VCF file containing information about each mutation was then output (Danecek et al. 2011). The hardcoded OTGsnpcaller pipeline was recoded, without any change to the function of the pipeline, to make it usable for this analysis. Analyses using this pipeline were then completed for the proband, parents, and siblings on a computational cluster located on campus.

\section{Variant Selection and Prioritization}

The resulting VCF file for each individual in each family was then converted into ANNOVAR (avinput) files using ANNOVAR software, as avinput files are easier to analyze because of their simpler format; avinput files provide information regarding chromosome number, start position, end position, reference nucleotide, alternate nucleotide, and quality scores for each variant (Wang et al. 2010). All avinput files for a particular family were then loaded into a Python program, which performs set intersections using DataFrame functions from the Pandas library, and set functions using the Numpy library to identify de novo and autosomal recessive variants (Van Der Walt et al. 2011). Autosomal recessive variants were identified by isolating homozygous variants in the affected child, intersecting these variants with variants that were heterozygous in both parents, and subtracting variants that were homozygous in the siblings. De novo variants were identified by subtracting variants found in the parents and sibling from variants found in the proband, using the following set functions performed using Pandas and Numpy libraries in Python:

$$
\begin{aligned}
\text { Autosomal recessive } & =\left[\left(M_{\text {het }} \cap F_{\text {het }}\right) \cap P_{\text {hom }}\right]-\mathrm{SIB}_{\text {hom }}, \\
\text { de novo } & =P_{\text {all }}-M_{\text {all }}-F_{\text {all }}-\mathrm{SIB}_{\text {all },}
\end{aligned}
$$

where $M$ refers to the mother's variants, $F$ refers to father's variants, $P$ refers to the proband's variants, and SIB refers to sibling's variants. The subscript het refers to heterozygous variants, hom refers to homozygous variants, and all refers to all variants.

The columns examined included the chromosome number, start point, end point, and zygosity of each called variant. The resulting avinput files were then output as BED files, which contain columns providing chromosome number, start point, and end point of the mutation. This process ensured that the resulting BED files contained all autosomal recessive and de novo variants that could be determined from the VCF. 
Using the GATK SelectVariants tool, these two BED files were intersected with the original VCF file two separate times, creating two VCF files, one containing only autosomal recessive variants and one containing only de novo variants. These files were then examined separately for all further analyses.

Both VCF files were then annotated with the Variant Effect Predictor (VEP) software (McLaren et al. 2010), which provided additional information about the variants. This annotated VCF file was then used with Genome Mining (GEMINI) software, which is a powerful, yet flexible network that allows for organization, sorting, and filtering of variants based on VEP and additional annotations. A Structured Query Language (SQL)-type database was created and loaded into GEMINI. After reviewing the GEMINI database schema to examine what annotations could be queried, factors were chosen based on three considerations: rarity, deleteriousness, and read quality. These factors were used as parameters during the GEMINI query to determine which variants would most likely be pathogenic.

Rarity was determined using the ExAC database, which contains population allele frequencies for exonic variants gathered from 60,706 unrelated individuals with no history of "severe pediatric disease" (Exome Aggregation Consortium et al. 2015). Variants were considered rare if not found in ExAC. Deleteriousness was mainly determined by CADD scores, which encompasses 63 annotations to determine a variant's deleteriousness. CADD scores are based on Phred quality scores; therefore, a minimum CADD score of 20, corresponding to the top $1 \%$ most deleterious variants, was selected as a cutoff (Kircher et al. 2014). Quality was determined using Phred scores for each variant. Although the resulting quality scores from the OTG-snpcaller pipeline did not correspond to the standard Phred quality score, a minimum cutoff score of 120 was decided after comparing variant calls with their corresponding BAM files. Chromosome number, start point, and end point columns of variants that met these three requirements were queried using GEMINI, and the output was saved as a BED file.

The BED file, along with Human Phenotype Ontology (HPO) terms corresponding to the proband's phenotype, was input into Phenolyzer, which aims to determine and prioritize which mutations contribute most to the phenotype by comparing the provided HPO terms to the phenotypes attributed to the gene in which the proband's mutation is located (Yang et al. 2015). A VCF file containing the same variants as the BED file used with Phenolyzer was then input into similar programs such as WANNOVAR and PhenIX in order to cross-reference several sources (Chang and Wang 2012; Zemojtel et al. 2014).

Additional deleteriousness scores for each variant of interest were also evaluated. SIFT and PolyPhen scores were evaluated for each variant to confirm its predicted deleteriousness. Variants with a SIFT score of $<0.05$ and a Polyphen score of $>0.995$ were considered deleterious.

\section{Confirmation of Variants}

Once a possible contributory mutation was identified, its location was then input into GoldenHelix GenomeBrowser, which displayed read information from the BAM files corresponding to each family. All variants of interest were researched thoroughly via PubMed searches and ruled out as major contributing mutations because of no association with a relevant phenotype.

The genic locations of each variant were identified using GEMINI. Initially, the known functions, phenotypes, and diseases associated with each gene were researched using the GeneCards online database, which contains information compiled from more than 100 sources (Rebhan et al. 1998; Stelzer et al. 2011; Dierking and Schmidtke 2014). These results were then confirmed by researching the gene in other databases, such as $\mathrm{NCBI}$, PubMed, and OMIM (Hamosh et al. 2005; Brown et al. 2015). These findings were also 
Competing Interest Statement

G.J.L. serves on advisory boards for GenePeeks, Inc. and Omicia, Inc. and has been a consultant to Good Start Genetics. K.W. is a board member and shareholder of Tute Genomics, Inc. R.R. is an employee, Chief Executive

Officer, and shareholder of Tute Genomics, Inc.

\section{Referees}

Anonymous

Keith K. Vaux

Received March 21, 2016; accepted in revised form July 13 , 2016. compared with the output of the phenotype analysis software. No additional contributing mutations were identified in this individual. The GEMINI query selected no autosomal recessive mutations of interest and 16 rare de novo mutations as mutations of interest. The SCN8A mutation was not found in the dbSNP nor in the 1000 Genomes databases.

\section{ADDITIONAL INFORMATION}

\section{Data Deposition and Access}

The sequencing data have been deposited to the NCBI Sequence Read Archive (SRA; http:// www.ncbi.nlm.nih.gov/sra) under accession number SRX1815960. The BioSample identifier is SAMN05187971. The mutation in SCN8A has been deposited to ClinVar (http://www.ncbi. nlm.nih.gov/clinvar/) under accession number SCV000282046.1.

\section{Ethics Statement}

Research was carried out in compliance with the Federal Policy for the Protection of Human Subjects 45C.F.R.46. The family was recruited to this study at the Utah Foundation for Biomedical Research (UFBR) where extensive clinical evaluation was performed. Written consent was obtained for phenotyping, use of facial photography, and WES through Protocol \#100 at the Utah Foundation for Biomedical Research, approved by the Independent Investigational Review Board, Inc.

\section{Acknowledgments}

G.J.L. is supported by funds from the Stanley Institute for Cognitive Genomics at Cold Spring Harbor Laboratory. K.W. is supported by National Institutes of Health (NIH) grant HG006465. M.H.M. is supported by NIH grant R01 NS34509. The authors thank Jacy Wagnon for preparation of Figure 4. The authors acknowledge Jason O'Rawe for bioinformatics support and comments on the manuscript. The authors thank the Exome Aggregation Consortium and the groups that provided exome-variant data for comparison. A full list of contributing groups can be found at http://ExAC.broadinstitute.org/about.

\section{REFERENCES}

Adzhubei I, Jordan DM, Sunyaev SR. 2013. Predicting functional effect of human missense mutations using PolyPhen-2. Curr Protoc Hum Genet Chapter 7: Unit7 20.

Bamshad MJ, Ng SB, Bigham AW, Tabor HK, Emond MJ, Nickerson DA, Shendure J. 2011. Exome sequencing as a tool for Mendelian disease gene discovery. Nat Rev Genet 12: 745-755.

Bergqvist AG, Schall JI, Stallings VA, Zemel BS. 2008. Progressive bone mineral content loss in children with intractable epilepsy treated with the ketogenic diet. Am J Clin Nutr 88: 1678-1684.

Blanchard MG, Willemsen MH, Walker JB, Dib-Hajj SD, Waxman SG, Jongmans MC, Kleefstra T, van de Warrenburg BP, Praamstra P, Nicolai J, et al. 2015. De novo gain-of-function and loss-of-function mutations of SCN8A in patients with intellectual disabilities and epilepsy. J Med Genet 52: 330-337.

Bragg LM, Stone G, Butler MK, Hugenholtz P, Tyson GW. 2013b. Shining a light on dark sequencing: characterising errors in lon Torrent PGM data. PLoS Comput Biol 9: e1003031.

Brown GR, Hem V, Katz KS, Ovetsky M, Wallin C, Ermolaeva O, Tolstoy I, Tatusova T, Pruitt KD, Maglott DR. 2015. Gene: a gene-centered information resource at NCBI. Nucleic Acids Res 43: D36-D42.

Chang X, Wang K. 2012. wANNOVAR: annotating genetic variants for personal genomes via the web. J Med Genet 49: 433-436.

Exome Aggregation Consortium, Lek M, Karczewski K, Minikel E, Samocha K, Banks E, Fennell T, O'DonnellLuria A, Ware J, Hill A, et al. 2015. Analysis of protein-coding genetic variation in 60,706 humans. bioRxiv doi: 10.1101/030338. 
Danecek P, Auton A, Abecasis G, Albers CA, Banks E, DePristo MA, Handsaker RE, Lunter G, Marth GT, Sherry ST, et al. 2011. The variant call format and VCFtools. Bioinformatics 27: 2156-2158.

de Kovel CG, Meisler MH, Brilstra EH, van Berkestijn FM, van 't Slot R, van Lieshout S, Nijman IJ, O'Brien JE, Hammer MF, Estacion M, et al. 2014. Characterization of a de novo SCN8A mutation in a patient with epileptic encephalopathy. Epilepsy Res 108: 1511-1518.

Dierking A, Schmidtke J. 2014. The future of Clinical Utility Gene Cards in the context of next-generation sequencing diagnostic panels. Eur J Hum Genet 22: 1247.

Dyment DA, Tetreault M, Beaulieu CL, Hartley T, Ferreira P, Warman Chardon J, Marcadier J, Sawyer SL, Mosca SJ, Micheil Innes A, et al. 2014. Whole-exome sequencing broadens the phenotypic spectrum of rare pediatric epilepsy: a retrospective study. Clin Genet 88: 34-40.

Estacion M, O'Brien JE, Conravey A, Hammer MF, Waxman SG, Dib-Hajj SD, Meisler MH. 2014. A novel de novo mutation of SCN8A $\left(\mathrm{Na}_{v} 1.6\right)$ with enhanced channel activation in a child with epileptic encephalopathy. Neurobiol Dis 69: 117-123.

Garrison E, Marth G. 2012. Haplotype-based variant detection from short-read sequencing. arXiv preprint arXiv:12073907.

Hamosh A, Scott AF, Amberger JS, Bocchini CA, McKusick VA. 2005. Online Mendelian Inheritance in Man (OMIM), a knowledgebase of human genes and genetic disorders. Nucleic Acids Res 33: D514D517.

Kahlig KM, Rhodes TH, Pusch M, Freilinger T, Pereira-Monteiro JM, Ferrari MD, van den Maagdenberg AM, Dichgans M, George AL Jr. 2008. Divergent sodium channel defects in familial hemiplegic migraine. Proc Natl Acad Sci 105: 9799-9804.

Kircher M, Witten DM, Jain P, O'Roak BJ, Cooper GM, Shendure J. 2014. A general framework for estimating the relative pathogenicity of human genetic variants. Nat Genet 46: 310-315.

Kong A, Frigge ML, Masson G, Besenbacher S, Sulem P, Magnusson G, Gudjonsson SA, Sigurdsson A, Jonasdottir A, Jonasdottir A, et al. 2012. Rate of de novo mutations and the importance of father's age to disease risk. Nature 488: 471-475.

Larsen J, Carvill GL, Gardella E, Kluger G, Schmiedel G, Barisic N, Depienne C, Brilstra E, Mang Y, Nielsen JE, et al. 2015. The phenotypic spectrum of SCN8A encephalopathy. Neurology 84: 480-489.

Lyon GJ, O'Rawe J. 2015. Human genetics and clinical aspects of neurodevelopmental disorders. In The genetics of neurodevelopmental disorders (ed. Mitchell K). Wiley, New York.

McKenna A, Hanna M, Banks E, Sivachenko A, Cibulskis K, Kernytsky A, Garimella K, Altshuler D, Gabriel S, Daly M. 2010. The Genome Analysis Toolkit: a MapReduce framework for analyzing next-generation DNA sequencing data. Genome Res 20: 1297-1303.

McLaren W, Pritchard B, Rios D, Chen Y, Flicek P, Cunningham F. 2010. Deriving the consequences of genomic variants with the Ensembl API and SNP Effect Predictor. Bioinformatics 26: 2069-2070.

Meisler MH, Helman G, Hammer MF, Fureman BE, Gaillard WD, Goldin AL, Hirose S, Ishii A, Kroner BL, Lossin C, et al. 2016. SCN8A encephalopathy: research progress and prospects. Epilepsia 57: 1027-1035.

Ng PC, Henikoff S. 2003. SIFT: predicting amino acid changes that affect protein function. Nucleic Acids Res 31: 3812-3814.

O'Brien JE, Meisler MH. 2013. Sodium channel SCN8A ( $\left.\mathrm{Na}_{\mathrm{v}} 1.6\right)$ : properties and de novo mutations in epileptic encephalopathy and intellectual disability. Front Genet 4: 213.

Ohba C, Kato M, Takahashi S, Lerman-Sagie T, Lev D, Terashima H, Kubota M, Kawawaki H, Matsufuji M, Kojima Y, et al. 2014. Early onset epileptic encephalopathy caused by de novo SCN8A mutations. Epilepsia 55: 994-1000.

O'Rawe J, Jiang T, Sun G, Wu Y, Wang W, Hu J, Bodily P, Tian L, Hakonarson H, Johnson WE, et al. 2013. Low concordance of multiple variant-calling pipelines: practical implications for exome and genome sequencing. Genome Med 5: 28.

O'Rawe JA, Ferson S, Lyon GJ. 2015. Accounting for uncertainty in DNA sequencing data. Trends Genet 31: 61-66.

Paila U, Chapman BA, Kirchner R, Quinlan AR. 2013. GEMINI: integrative exploration of genetic variation and genome annotations. PLoS Comput Biol 9: e1003153.

Rebhan M, Chalifa-Caspi V, Prilusky J, Lancet D. 1998. GeneCards: a novel functional genomics compendium with automated data mining and query reformulation support. Bioinformatics 14: 656-664.

Richards S, Aziz N, Bale S, Bick D, Das S, Gastier-Foster J, Grody WW, Hegde M, Lyon E, Spector E, et al. 2015. Standards and guidelines for the interpretation of sequence variants: a joint consensus recommendation of the American College of Medical Genetics and Genomics and the Association for Molecular Pathology. Genet Med 17: 405-424.

Salipante SJ, Kawashima T, Rosenthal C, Hoogestraat DR, Cummings LA, Sengupta DJ, Harkins TT, Cookson BT, Hoffman NG. 2014. Performance comparison of Illumina and ion torrent next-generation sequencing platforms for $16 \mathrm{~S}$ rRNA-based bacterial community profiling. Appl Environ Microbiol 80: 7583-7591. 
Stelzer G, Dalah I, Stein TI, Satanower Y, Rosen N, Nativ N, Oz-Levi D, Olender T, Belinky F, Bahir I. 2011. Insilico human genomics with GeneCards. Hum Genomics 5: 709-717.

Vaher U, Noukas M, Nikopensius T, Kals M, Annilo T, Nelis M, Ounap K, Reimand T, Talvik I, Ilves P, et al. 2014. De novo SCN8A mutation identified by whole-exome sequencing in a boy with neonatal epileptic encephalopathy, multiple congenital anomalies, and movement disorders. J Child Neurol 29: NP202-NP206.

Van Der Walt S, Colbert SC, Varoquaux G. 2011. The NumPy array: a structure for efficient numerical computation. Comput Sci Eng 13: 22-30.

Veeramah KR, O'Brien JE, Meisler MH, Cheng X, Dib-Hajj SD, Waxman SG, Talwar D, Girirajan S, Eichler EE, Restifo LL, et al. 2012. De novo pathogenic SCN8A mutation identified by whole-genome sequencing of a family quartet affected by infantile epileptic encephalopathy and SUDEP. Am J Hum Genet 90: 502-510.

Wagnon JL, Meisler MH. 2015. Recurrent and non-recurrent mutations of SCN8A in epileptic encephalopathy. Front Neurol 6: 104.

Wagnon JL, Barker BS, Hounshell JA, Haaxma CA, Shealy A, Moss T, Parikh S, Messer RD, Patel MK, Meisler MH. 2016. Pathogenic mechanism of recurrent mutations of SCN8A in epileptic encephalopathy. Ann Clin Transl Neurol 3: 114-123.

Wang K, Li M, Hakonarson H. 2010. ANNOVAR: functional annotation of genetic variants from high-throughput sequencing data. Nucleic Acids Res 38: e164.

Yang H, Robinson PN, Wang K. 2015. Phenolyzer: phenotype-based prioritization of candidate genes for human diseases. Nat Methods 12: 841-843.

Zemojtel T, Köhler S, Mackenroth L, Jäger M, Hecht J, Krawitz P, Graul-Neumann L, Doelken S, Ehmke N, Spielmann M. 2014. Effective diagnosis of genetic disease by computational phenotype analysis of the disease-associated genome. Sci Transl Med 6: 252ra123.

Zhu P, He L, Li Y, Huang W, Xi F, Lin L, Zhi Q, Zhang W, Tang YT, Geng C, et al. 2014. OTG-snpcaller: an optimized pipeline based on TMAP and GATK for SNP calling from ion torrent data. PLoS One 9: e97507. 


\section{COLD SPRING HARBOR Molecular Case Studies}

\section{SCN8A mutation in a child presenting with seizures and developmental delays}

Janet Malcolmson, Robert Kleyner, David Tegay, et al.

Cold Spring Harb Mol Case Stud 2016, 2: a001073 originally published online July 19, 2016 Access the most recent version at doi: $10.1101 / \mathrm{mcs} . a 001073$
Supplementary http://molecularcasestudies.cshlp.org/content/suppl/2016/07/19/mcs.a001073.D Material C1
References This article cites 40 articles, 8 of which can be accessed free at: http://molecularcasestudies.cshlp.org/content/2/6/a001073.full.html\#ref-list-1
License This article is distributed under the terms of the Creative Commons Attribution License, which permits unrestricted reuse and redistribution provided that the original author and source are credited.
Email Alerting Receive free email alerts when new articles cite this article - sign up in the box at the Service top right corner of the article or click here.

